\title{
Electron transport analysis in water vapor
}

\author{
Satoru Kawaguchi ${ }^{1}$, Kazuhiro Takahashi², Kohki Satoh ${ }^{2 *}$, and Hidenori Itoh ${ }^{2}$ \\ ${ }^{1}$ Division of Engineering, Graduate School of Muroran Institute of Technology, Muroran, Hokkaido 050-8585, Japan \\ ${ }^{2}$ Division of Information and Electronic Engineering, Graduate School of Muroran Institute of Technology, Muroran, Hokkaido 050-8585, Japan \\ *E-mail: ksatoh@mmm.muroran-it.ac.jp \\ Received November 30, 2015; revised January 21, 2016; accepted January 30, 2016; published online xxxx yy, zzzz
}

A reliable set of electron collision cross sections for water vapor, including elastic, rotational, vibrational, and electronic excitation, electron attachment, and ionization cross sections, is estimated by the electron swarm method. In addition, anisotropic electron scattering for elastic and rotational excitation collisions is considered in the cross section set. Electron transport coefficients such as electron drift velocity, longitudinal diffusion coefficient, and effective ionization coefficient are calculated from the cross section set by Monte Carlo simulation in a wide range of $E / N$ values, where $E$ and $N$ are the applied electric field and the number density of $\mathrm{H}_{2} \mathrm{O}$ molecules, respectively. The calculated transport coefficients are in good agreement with those measured. The obtained results confirm that the anisotropic electron scattering is important for the calculation at low $E / N$ values. Furthermore, the cross section set assuming the isotropic electron scattering is proposed for practical use.

(C) 2016 The Japan Society of Applied Physics

\section{Introduction}

Discharge plasma, generated in gases containing water vapor or generated in contact with liquid, has extensive applications, such as the promotion of plant growth, ${ }^{1)}$ the disinfection of microorganisms, ${ }^{2-4)}$ and the decomposition of nondegradable hazardous substances. ${ }^{5,6)}$ Reactive nitrogen species (RNS), such as $\mathrm{NO}_{3}{ }^{-}$, and reactive oxygen species (ROS), such as $\mathrm{H}_{2} \mathrm{O}_{2}$, are found to play important roles in these applications; thus, an appropriate method of controlling the concentrations of these species is needed to improve the efficiency of the applications. Plasma simulation is known as an effective tool for predicting the behavior of active species in plasmas, and such simulation has been carried out. ${ }^{7-9)}$

The reliability of plasma simulation coupled with rate equation analysis for chemical reaction processes depends on that of transport coefficients, such as a drift velocity and diffusion coefficient, and rate coefficients for species. In particular, electrons have high mobility and reactivity, so that accurate transport coefficients and rate coefficients for electrons are needed to improve the accuracy of plasma simulation. The transport coefficients for electrons are not only measured but also calculated from a set of electron 42 collision cross sections by Monte Carlo simulation and 43 Boltzmann equation analysis. Furthermore, measured trans44 port coefficients are limited, so the calculation of transport 45 coefficients from an accurate cross section set is important; 46 therefore, an accurate cross section set is required.

47 Some cross section sets for water vapor have been 48 reported. ${ }^{10-12)}$ Itikawa and Mason ${ }^{10)}$ reviewed measured and 49 theoretically calculated electron collision cross sections for 50 water vapor, and recommended a set of cross sections 51 including momentum transfer, rotational, vibrational, and 52 electronic excitation, electron attachment, and ionization 53 cross sections, but they did not calculate the electron 54 transport coefficients from their cross section set; therefore, 55 the validity of the cross section set is not confirmed. Yousfi ${ }_{56}$ and Benabdessadok ${ }^{11)}$ and de Urquijo et al. ${ }^{12)}$ estimated the 57 cross section set for water vapor by the electron swarm 58 method with Boltzmann equation analysis. In the calculation 59 of the transport coefficients, the effect of superelastic so collision for rotationally excited molecules is considered 61 by Yousfi and Benabdessadok, ${ }^{11)}$ and the effect of the superelastic collision and anisotropic electron scattering for 19 elastic and rotational excitation collisions are considered 20 by de Urquijo et al.; ${ }^{12)}$ however, the electron drift velocity, 21 longitudinal diffusion coefficient, and effective ionization 22 coefficient calculated from the cross section sets in Refs. $11_{23}$ and 12 do not necessarily agree with measured data in a wide 24 range of $E / N$ values, where $E$ and $N$ are the applied electric 25 field and the number density of $\mathrm{H}_{2} \mathrm{O}$ molecules, respectively. 26

In this work, we estimated the reliable cross section set 27 for water vapor considering the anisotropic electron scattering 28 for elastic and rotational excitation collisions. The electron 29 transport coefficients such as the electron drift velocity, 30 longitudinal diffusion coefficient, and effective ionization 31 coefficient are calculated from the estimated cross section set 32 by Monte Carlo simulation. Then, the validity of the estimated 33 cross section set is confirmed by the comparison between 34 calculated and measured transport coefficients. Furthermore, 35 a set of electron collision cross sections for water vapor 36 assuming isotropic electron scattering after elastic and ${ }_{37}$ rotational excitation collisions is estimated for practical use. 38

\section{Electron collision cross sections (anisotropic scattering model)}

Figure 1 shows the electron collision cross section set for 42 water vapor estimated in this work. This cross section set 43 consists of two vibrational excitations, three electron attach- 44 ments, 26 electronic excitations, six ionizations, three 45 rotational excitation cross sections, and one elastic collision 46 cross section. Electron-molecule reactions considered here 47 are listed in Table I. Symbol $\Sigma q_{\text {ex }}$ in Fig. 1 denotes the sum 48 of electronic excitation cross sections, and the detailed cross 49 sections for the electronic excitation are plotted in Fig. 2. 50 The vibrational excitation cross sections follow experimental 51 data obtained by Seng and Linder. ${ }^{13)}$ For the electron 52 attachment, partial cross sections for $\mathrm{O}^{-}$and $\mathrm{H}^{-}$measured 53 by Rawat et al. ${ }^{14)}$ and that for $\mathrm{OH}^{-}$measured by Melton ${ }^{15)}{ }_{54}$ are used. For the electronic excitation, 25 out of 26 partial 55 cross sections follow those measured by Thorn and co- 56 workers, ${ }^{16-20)}$ and a cross section, the threshold energy of 57 which is $12.5 \mathrm{eV}$, is estimated to fit the calculated effective 58 ionization coefficient to the measured data. The ionization 59 cross section consists of five partial dissociative ionization 60 cross sections such as $\mathrm{OH}^{+}, \mathrm{H}^{+}, \mathrm{O}^{+}, \mathrm{H}_{2}^{+}$, and $\mathrm{O}^{2+}$ and a 61 


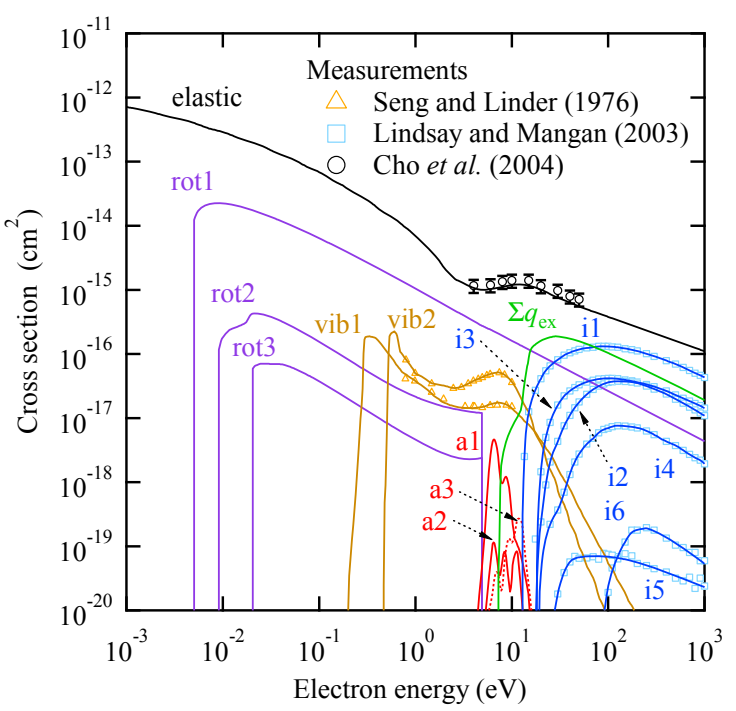

Fig. 1. (Color online) Electron collision cross sections of water vapor (anisotropic scattering model).

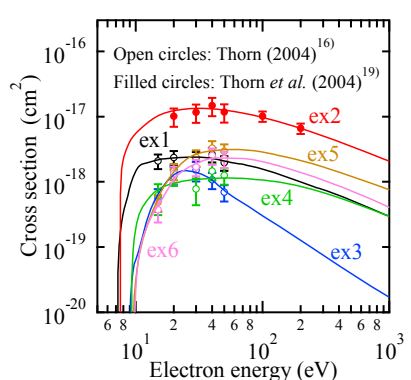

(a)

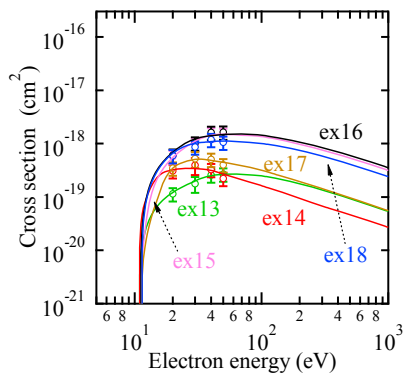

(c)

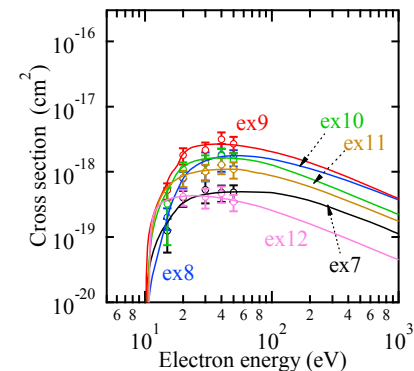

(b)

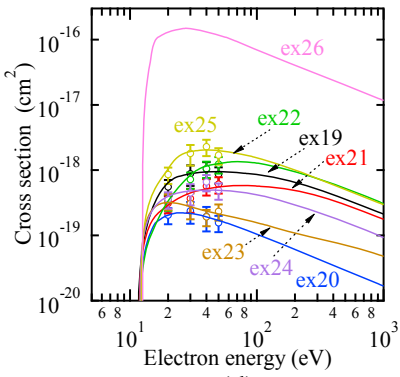

(d)
Fig. 2. (Color online) Electronic excitation cross sections: (a) ex1-ex6, (b) ex7-ex12, (c) ex13-ex18, and (d) ex19-ex26.

direct ionization cross section $\mathrm{H}_{2} \mathrm{O}^{+}$, and those follow the data recalibrated by Lindsay and Mangan ${ }^{21)}$ using the 48 measured data of Straub et al. ${ }^{22)}$ The threshold energies of 49 the ionization cross sections for $\mathrm{H}_{2} \mathrm{O}^{+}, \mathrm{OH}^{+}$and $\mathrm{H}^{+}, \mathrm{O}^{+}$, and $50 \mathrm{H}_{2}{ }^{+}$are obtained from the measured data by Snow and ${ }^{51}$ Thomas, ${ }^{23)}$ Lefaivre and Marmet, ${ }^{24)}$ Morrison and Traeger, ${ }^{25)}$ 52 and Ehrhardt and Kresling ${ }^{26)}$ listed in NIST Chemistry ${ }_{53}$ WebBook ${ }^{27)}$ respectively. The threshold energy of the cross ${ }^{54}$ section for $\mathrm{O}^{2+}$ is estimated as $80 \mathrm{eV}$ by the extrapolation of 55 the measured partial dissociative ionization cross section. ${ }_{56}$ Rotational excitation cross sections have been theoretically 57 calculated owing to experimental difficulty. Itikawa and ${ }_{58}$ Mason $^{10)}$ reported the rotational excitation cross sections for 59 the transitions from $J=0$ to 1,2 , and 3 based on the data so recently calculated by Faure et al. ${ }^{28)}$ where $J$ is the rotational 61 angular momentum. In this work, the recent and recom-
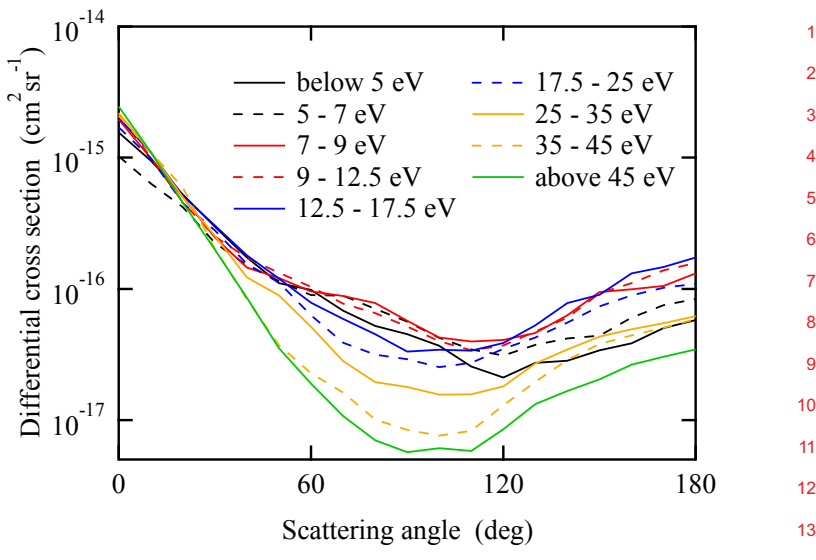

Fig. 3. (Color online) Differential cross sections for elastic and rotational ${ }_{15}$ excitation collisions.

mended cross sections by Itikawa and Mason are used, but 18 multiplied by a factor of 0.1 . The vibrationally elastic cross 19 section, which is defined as the sum of the elastic and 20 rotational excitation cross sections, is measured by Cho 21 et al. ${ }^{29)}$ so that the elastic collision cross section is estimated 22 from the data.

Figure 3 shows differential cross sections for elastic and 24 rotational excitation collisions for different incident energies 25 deduced from the data measured by Cho et al. ${ }^{29)}$ The angular 26 distributions are used after elastic and rotational excitation 27 collisions.

\section{Monte Carlo simulation}

The behavior of electrons in free space under a uniform 31 electric field $\boldsymbol{E}=(0,0,-E)$ is traced by the Monte Carlo 32 method, and electron transport coefficients such as the mean- 33 arrival-time drift velocity $W_{\mathrm{m}}$ and longitudinal diffusion ${ }_{34}$ coefficient $D_{\mathrm{L}}$ for time-of-flight (TOF) sampling, the ioniza- 35 tion coefficient $\alpha$, electron attachment coefficient $\eta$, and ${ }_{36}$ effective ionization coefficient $\alpha-\eta$ for steady-state Town- 37 send (SST) sampling are obtained. The definition and 38 sampling principle of those transport coefficients are describ- 39 ed in Refs. 30 and 31 . The drift velocity $W_{\mathrm{m}}$ is related to the 40 center-of-mass drift velocity $W_{\mathrm{r}}$, which is also called the bulk 41 drift velocity, ${ }^{32)}$ and the relationship is described as ${ }^{33)} \quad 42$

$$
W_{\mathrm{m}}=W_{\mathrm{r}}-2(\alpha-\eta) D_{\mathrm{L}}+3(\alpha-\eta)^{2} D_{3}-\cdots, \quad \text { (1) }{ }^{43}
$$

where $D_{3}$ is the third-order diffusion coefficient in the field 45 direction. The drift velocity $W_{\mathrm{m}}$ differs from the drift velocity 46 $W_{\mathrm{r}}$, and the electron drift velocity measured by the double- 47 shutter drift tube method ${ }^{34)}$ and pulsed experiment ${ }^{12)}$ should 48 be compared with $W_{\mathrm{m}} \cdot{ }^{35}$ ) The calculation method of Monte 49 Carlo simulation for electron behavior is described in 50 Refs. 30 and 31.

The thermal motion of molecules and the anisotropic 52 electron scattering are reported as important phenomena for 53 the analysis of electron behavior in water vapor in a low $E / N_{54}$ region. For the accurate simulation of electron behavior, the 55 velocity $\boldsymbol{v}^{\prime}$ of an electron after a collision is calculated as ${ }^{36)}{ }_{56}$

$$
\boldsymbol{v}^{\prime}=\frac{M}{m+M} \boldsymbol{g}^{\prime}+\frac{m \boldsymbol{v}+M \boldsymbol{V}}{m+M},
$$

where $m$ and $M$ are the masses of the electron and molecule, 60 respectively, $\boldsymbol{V}=\left(V_{x}, V_{y}, V_{z}\right)$ and $\boldsymbol{V}^{\prime}$ are the velocities of the 61 
Table I. Electron-water molecule reactions considered in the estimated cross section set.

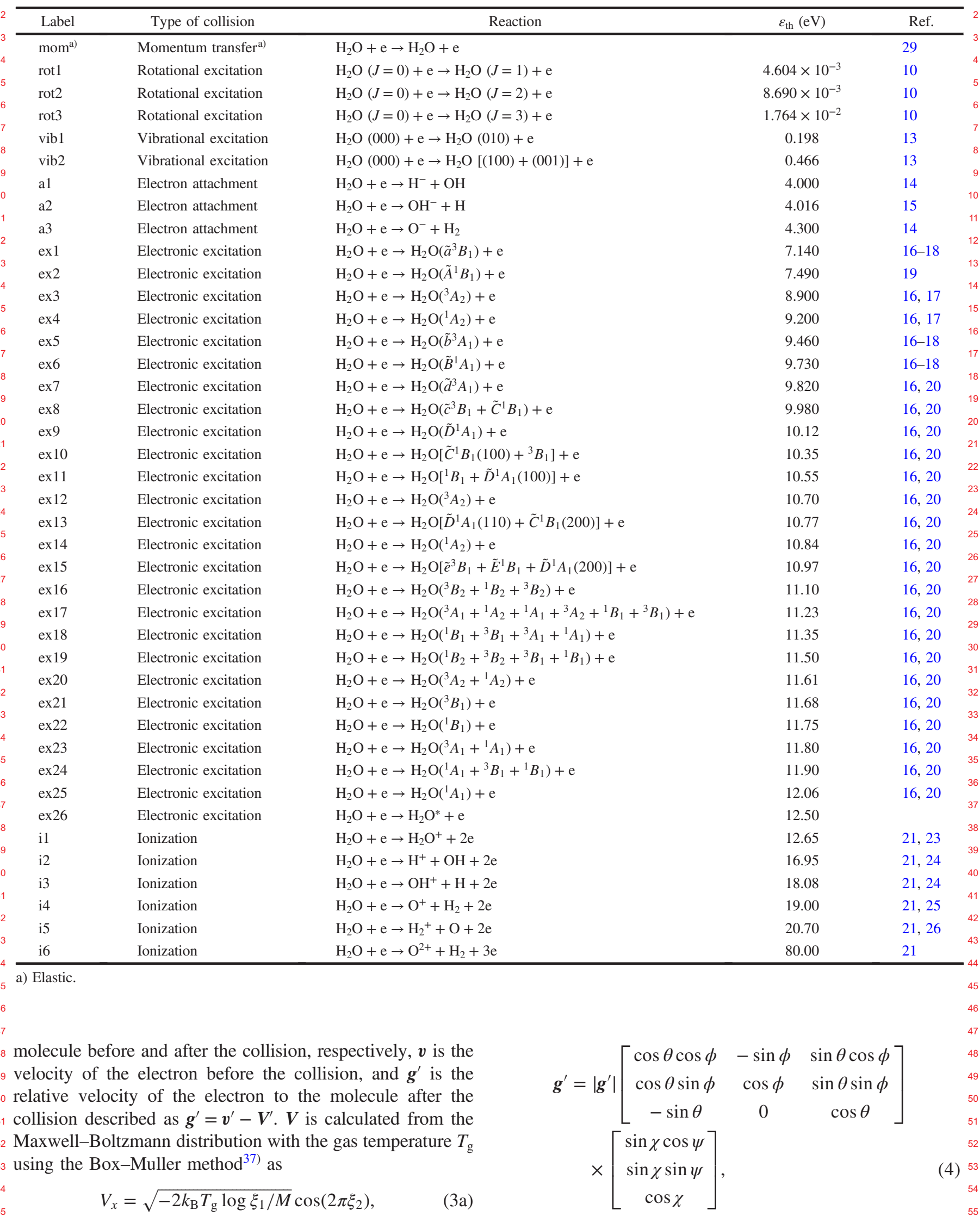

$$
\begin{aligned}
& V_{y}=\sqrt{-2 k_{\mathrm{B}} T_{\mathrm{g}} \log \xi_{1} / M} \sin \left(2 \pi \xi_{2}\right), \\
& V_{z}=\sqrt{-2 k_{\mathrm{B}} T_{\mathrm{g}} \log \xi_{3} / M} \cos \left(2 \pi \xi_{4}\right),
\end{aligned}
$$

where $k_{\mathrm{B}}$ is the Boltzmann constant and $\xi$ is the random so number uniformly distributed on $[0,1]$. The relative velocity ${ }_{61} \boldsymbol{g}^{\prime}$ is calculated as where $\theta$ and $\phi$ are respectively the polar and azimuthal angles 56 of the relative velocity $\boldsymbol{g}=\boldsymbol{v}-\boldsymbol{V}$ in a laboratory system, and 57 the scattering angles $\chi$ and $\psi$ are respectively the polar and 58 azimuthal angles of $\boldsymbol{g}^{\prime}$ in the coordinate system where $\boldsymbol{g}$ is 59 directed along the $z$-axis as shown in Fig. $4{ }^{38)}$ The scattering 60 angle $\chi$ is obtained from Eq. (5), and $\psi$ from Eq. (6), 


$$
\begin{aligned}
\xi_{5} & =\int_{0}^{\chi} q\left(\varepsilon, \theta^{\prime}\right) \sin \theta^{\prime} d \theta^{\prime} / \int_{0}^{\pi} q\left(\varepsilon, \theta^{\prime}\right) \sin \theta^{\prime} d \theta^{\prime}, \\
\psi & =2 \pi \xi_{6},
\end{aligned}
$$

where $q\left(\varepsilon, \theta^{\prime}\right)$ is the differential cross section at the energy $\varepsilon$ and angle $\theta^{\prime}$. When electron scattering is assumed to be isotropic, the scattering angle $\chi$ can be calculated as

$$
\cos \chi=1-2 \xi_{5} \text {. }
$$

The speed for relative velocity after collision $\left|\boldsymbol{g}^{\prime}\right|$ is obtained from the principle of the conservation of energy. For elastic collision, the total kinetic energy of colliding molecule and electron is conserved, and $\left|\boldsymbol{g}^{\prime}\right|$ is equal to $|\boldsymbol{g}|$. For inelastic collision, $\left|\boldsymbol{g}^{\prime}\right|$ is calculated as

$$
\left|\boldsymbol{g}^{\prime}\right|=\sqrt{|\boldsymbol{g}|^{2}-\frac{2}{\mu} \varepsilon_{\mathrm{th}}},
$$

where $\mu$ is the reduced mass described as $\mu=m M /(m+M)$ and $\varepsilon_{\text {th }}$ is the threshold energy for the collision. For superelastic collision, which is the inverse process of the excitation collision having the threshold energy $\varepsilon_{\mathrm{th}},\left|\boldsymbol{g}^{\prime}\right|$ is calculated as

$$
\left|\boldsymbol{g}^{\prime}\right|=\sqrt{|\boldsymbol{g}|^{2}+\frac{2}{\mu} \varepsilon_{\mathrm{th}} .}
$$

The number density $N$ is $3.535 \times 10^{16} \mathrm{~cm}^{-3}$ at $0{ }^{\circ} \mathrm{C}$ and 1 Torr, and the collision between an electron and a $\mathrm{H}_{2} \mathrm{O}$ molecule is only considered, assuming the low ionization degree in a typical swarm experiment. The populations of rotational excitation states for thermally excited molecules are neglected because of a lack of reliable data for rotational excitation

\section{Results and discussion}

44 4.1 Comparison between measured and calculated 45 transport coefficients

${ }_{46}$ Figure 5 shows the values of mean-arrival-time drift velocity ${ }_{47} W_{\mathrm{m}}$ in water vapor calculated from the present cross sec48 tion set as a function of $E / N$, together with those calculated 49 from the cross section sets reported by Yousfi and 50 Benabdessadok, ${ }^{11)}$ Itikawa and Mason, ${ }^{10)}$ and de Urquijo 51 et al. ${ }^{12)}$ The values of $W_{\mathrm{m}}$ measured by the double-shutter 52 drift tube method ${ }^{34)}$ and pulsed experiment ${ }^{12)}$ are also plotted. 53 The calculated values from the Yousfi and Benabdessadok's 54 cross section set ${ }^{11)}$ are close to the measured data below ${ }_{55} E / N=40 \mathrm{Td}$ and above $E / N=160 \mathrm{Td}$, but they do not agree 56 with the measured data between 40 and $160 \mathrm{Td}$. Although 57 the calculated values from Itikawa and Mason's cross section 58 set $^{10)}$ disagree with the measured data, the values of $W_{\mathrm{m}}$ 59 calculated from the cross section sets estimated here and 60 reported by de Urquijo et al. ${ }^{12)}$ are found to agree well with 61 the measured data.

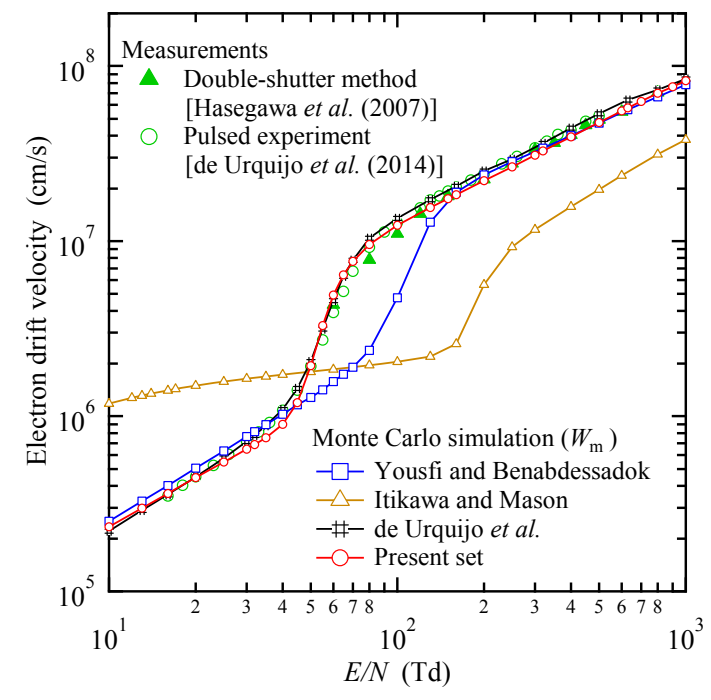

Fig. 5. (Color online) Electron drift velocity in water vapor as a function 18 of $E / N$.

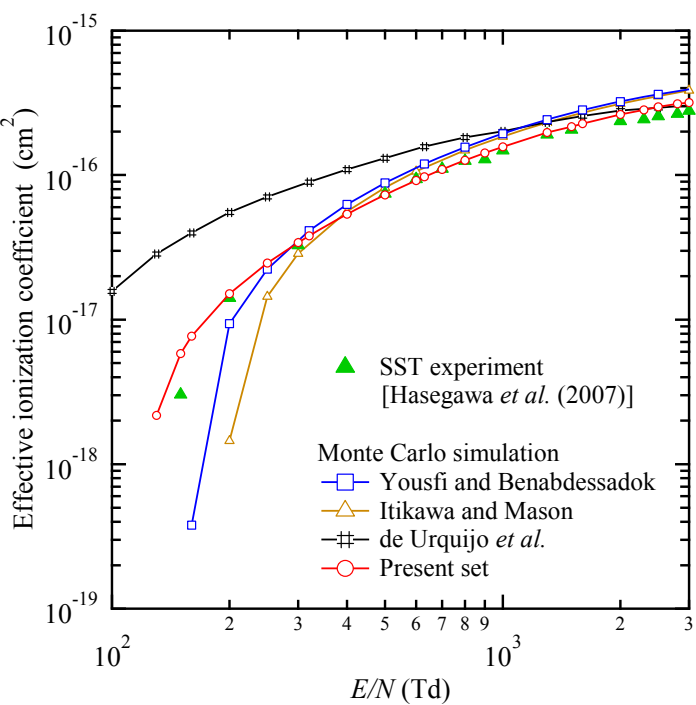

20

Fig. 6. (Color online) Effective ionization coefficient in water vapor as a function of $E / N$.

Figure 6 shows the values of effective ionization coef- 42 ficient $(\alpha-\eta) / N$ in water vapor calculated from the present 43 cross section set as a function of $E / N$, together with those 44 calculated from the previously reported cross section 45 sets ${ }^{10-12)}$ and measured by steady-state Townsend experi- 46 ment. $^{34)}$ The $(\alpha-\eta) / N$ values calculated from the cross 47 section set reported by de Urquijo et al. ${ }^{12)}$ are larger than the 48 measured data in a wide range of $E / N$ values. The values 49 calculated from the other reported cross section sets ${ }^{10,11)}$ are 50 close to the measured data between 300 and $800 \mathrm{Td}$, but 51 disagree with the measured data in the other $E / N$ region. The 52 values calculated from the present cross section set agree 53 with the measured data within the difference of $10 \%$ between 54 200 and $2,000 \mathrm{Td}$, and within the difference of $15 \%$ above 55 $E / N=2,000 \mathrm{Td}$. The difference in $(\alpha-\eta) / N$ above 2,000 Td 56 may affect the ionization growth of electrons. Although the 57 difference between the calculated value and measured data is 58 found to be $48 \%$ at $E / N=150 \mathrm{Td},(\alpha-\eta) / N$ is quite small; 59 therefore, the influence of the difference in $(\alpha-\eta) / N$ on the 60 ionization growth of electrons can be ignored. 


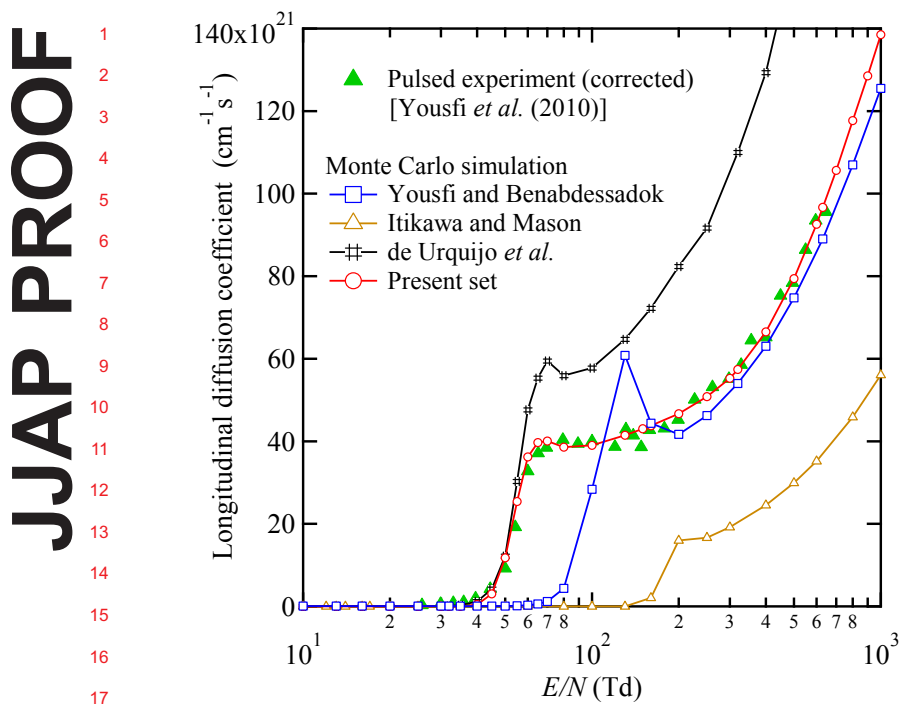

Fig. 7. (Color online) Longitudinal diffusion coefficient in water vapor as a function of $E / N$

Figure 7 shows the values of longitudinal diffusion coefficient $N D_{\mathrm{L}}$ in water vapor calculated from the present cross section set as a function of $E / N$, together with those calculated from the previously reported cross section sets ${ }^{10-12)}$ and measured by pulsed experiment. ${ }^{39)}$ Here, the ${ }_{27}$ values of $N D_{\mathrm{L}}$ measured by pulsed experiment are corrected ${ }_{28}$ in accordance with the relation with $N D_{\mathrm{L}}$ measured by TOF 29 experiment reported by Terashita et al. ${ }^{40}$ ) The values of $N D_{\mathrm{L}}$ calculated from Yousfi and Benabdessadok's set ${ }^{11)}$ are close to the measured data above $E / N=200 \mathrm{Td}$, but disagree 32 with the data below $E / N=160 \mathrm{Td}$. Those calculated from 33 the other reported cross section sets ${ }^{10,12)}$ disagree with the ${ }_{34}$ measured data. The $N D_{\mathrm{L}}$ values calculated from the present 35 cross section set agree well with the measured data.

The electron transport coefficients calculated from the present cross section set agree well with the measured data in 38 a wide range of $E / N$ values, and this confirms the validity of 39 the present cross section set.

40

\subsection{Influences of superelastic collision on transport 2 properties}

${ }_{43}$ Since superelastic collision for rotationally excited molecules 44 may affect the transport properties of electrons in water 45 vapor, especially at low $E / N$ values, the superelastic collision 46 has been considered in several studies. ${ }^{11,12,41)}$ Thus, the 47 influences of the superelastic collision on the electron 48 transport coefficients in water vapor are examined. Figure 8 49 shows the superelastic collision cross sections $q_{J^{\prime} \rightarrow 0}$ for the 50 transition from $J=J^{\prime}$ to 0 calculated from the following ${ }_{51}$ equation derived from the principle of detailed balance: ${ }^{36)}$

$$
q_{J^{\prime} \rightarrow 0}(\varepsilon)=\frac{1}{2 J^{\prime}+1} \frac{\varepsilon+\varepsilon_{\mathrm{th}}}{\varepsilon} q_{0 \rightarrow J^{\prime}}\left(\varepsilon+\varepsilon_{\mathrm{th}}\right),
$$

55 where $q_{0 \rightarrow J^{\prime}}$ is the rotational excitation cross section for the ${ }_{56}$ transition from $J=0$ to $J^{\prime}$ and $\varepsilon_{\text {th }}$ is the threshold energy of 57 $q_{0 \rightarrow J^{\prime}}$. The scattering angle after the superelastic collision is ${ }_{58}$ calculated using the differential cross sections shown in 59 Fig. 3.

Figure 9 shows the values of $W_{\mathrm{m}},(\alpha-\eta) / N$, and $N D_{\mathrm{L}}$ ${ }_{61}$ calculated from the cross section set considering the super-

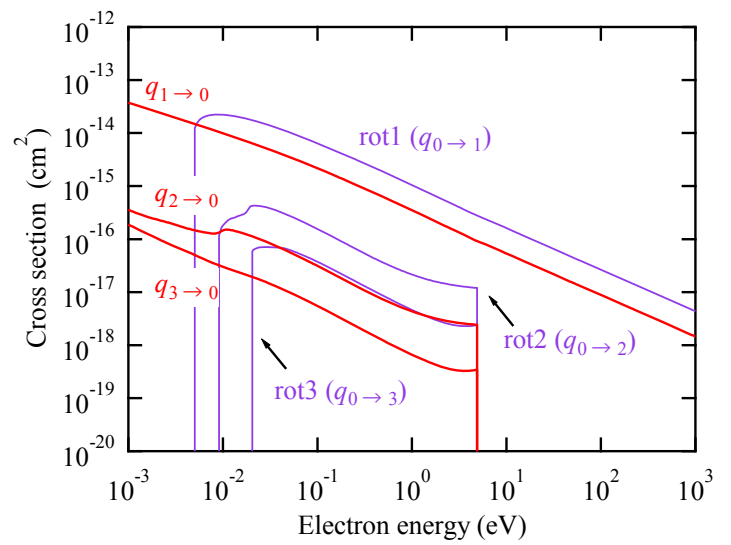

Fig. 8. (Color online) Superelastic collision cross sections for water vapor.

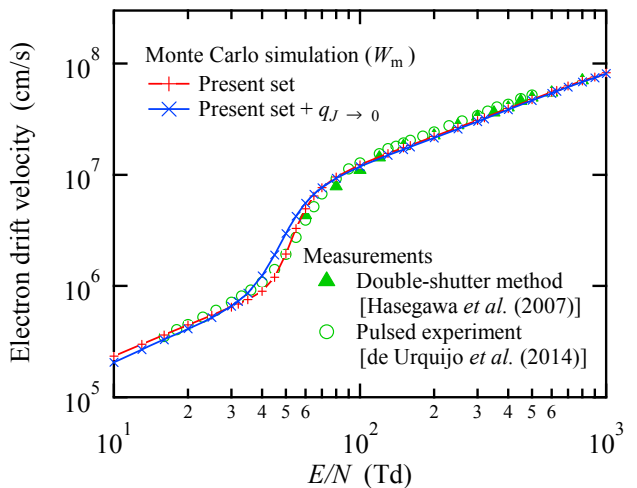

(a)

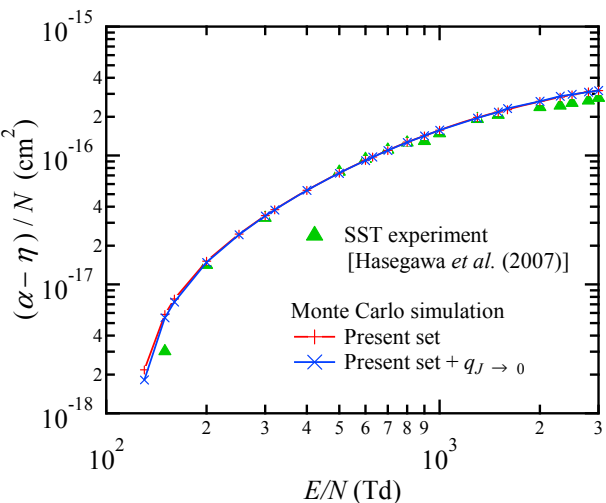

(b)

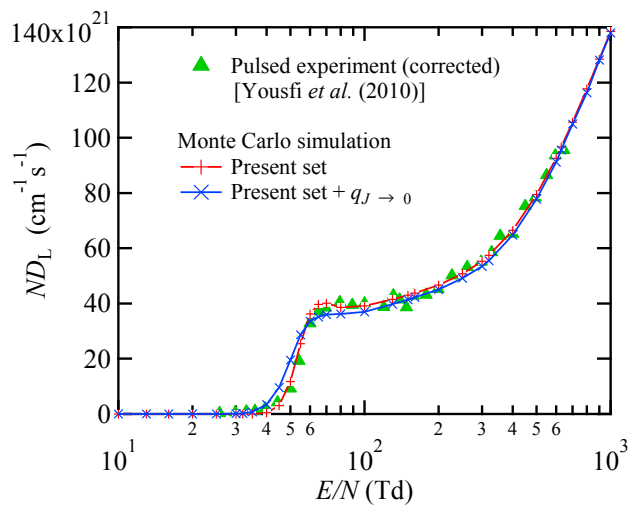

(c)

Fig. 9. (Color online) Influence of superelastic collision on the electron transport coefficients in water vapor: (a) electron drift velocity, (b) effective 60 ionization coefficient, and (c) longitudinal diffusion coefficient. 


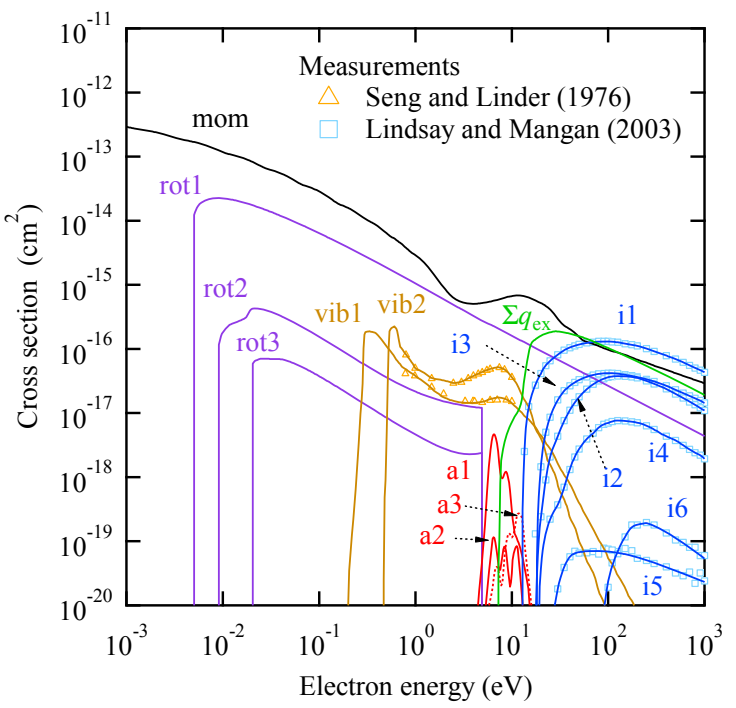

Fig. 10. (Color online) Electron collision cross sections of water vapor assuming the isotropic electron scattering (isotropic scattering model).

elastic collision as functions of $E / N$. The slight influence of superelastic collision on $W_{\mathrm{m}}$ and $N D_{\mathrm{L}}$ is found below $E / N=100 \mathrm{Td}$, but no significant difference is found in ${ }_{25}(\alpha-\eta) / N$. This indicates that the influence of superelastic 26 collision is negligible and limited.

\section{${ }_{28} 4.3$ Isotropic scattering model for practical use}

${ }_{29}$ In the calculation of electron transport coefficients and rate 30 coefficients using electron collision cross sections, it is not 31 necessarily easy to consider the anisotropic electron scatter32 ing. Moreover, those coefficients are often calculated by a 33 two-term approximation Boltzmann equation solver like ${ }_{34}$ BOLSIG $+{ }^{42}$ which assumes electron scattering to be 35 isotropic. For practical use, the electron collision cross 36 section set for water vapor assuming the isotropic electron 37 scattering is therefore estimated. Figure 10 shows the 38 estimated cross section set called the isotropic scattering 39 model. The elastic collision cross section in Fig. 1 is replaced 40 with the elastic momentum transfer cross section shown in ${ }_{41}$ Fig. 10. The other cross sections are the same as those in 42 Figs. 1 and 2.

43 Figure 11 shows the values of the electron transport 44 coefficients in water vapor calculated by Monte Carlo simu-

45 lation using the cross section sets shown in Fig. 1 (aniso-

46 tropic scattering model) and Fig. 10 (isotropic scattering 47 model) as functions of $E / N$, together with the measured 48 data. ${ }^{12,34,39)}$ It is found that the transport coefficients cal49 culated from the cross section set assuming the isotropic 50 electron scattering agree with those calculated from the cross 51 section set considering the anisotropic electron scattering.

52 Figures 12 and 13 show rate coefficients such as $\Sigma k_{\text {rot }}$, ${ }_{53} \Sigma k_{\text {vib }}, \Sigma k_{\mathrm{a}}, \Sigma k_{\mathrm{ex}}$, and $\Sigma k_{\mathrm{i}}$ and those concerning $\mathrm{OH}$ radical 54 generation in water vapor calculated from the two cross 55 section sets as functions of $E / N$, respectively. Symbols $\Sigma k_{\text {rot }}$, ${ }_{56} \Sigma k_{\text {vib }}, \Sigma k_{\mathrm{a}}, \Sigma k_{\mathrm{ex}}$, and $\Sigma k_{\mathrm{i}}$ in Fig. 12 denote the sum of rate 57 coefficients for rotational excitation, vibrational excitation, 58 electron attachment, electronic excitation, and ionization 59 collisions, respectively. The rate coefficients calculated from 60 the two cross section sets agree very well. This indicates 61 that the cross section set assuming the isotropic electron

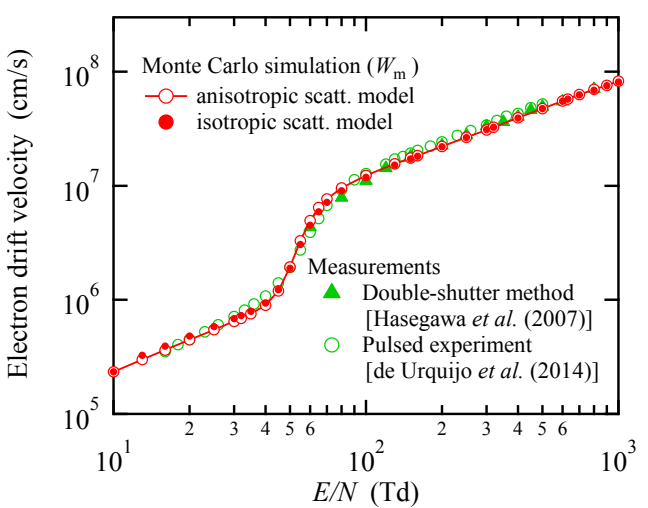

(a)

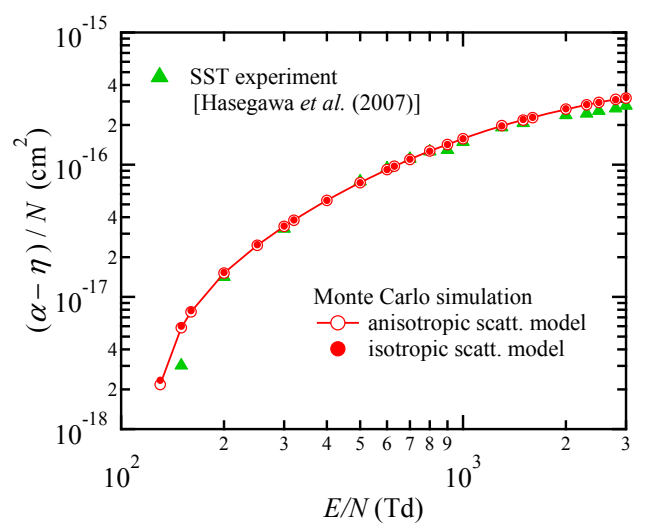

(b)

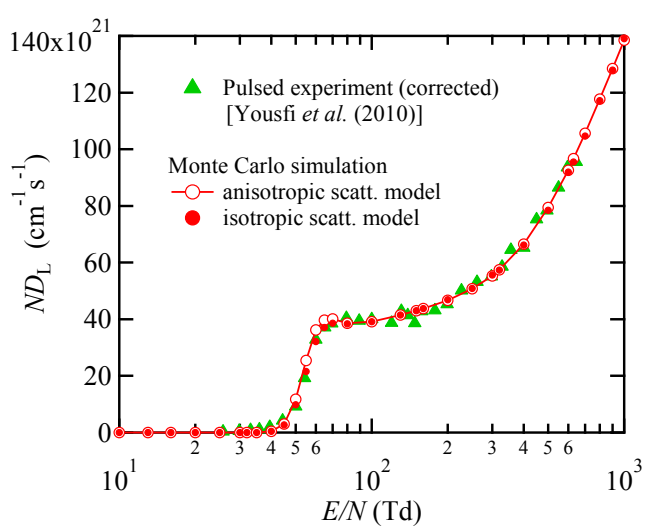

(c)

Fig. 11. (Color online) Comparison between the electron transport coefficients calculated from the two cross section sets: (a) electron drift velocity, (b) effective ionization coefficient, and (c) longitudinal diffusion coefficient.

scattering can be used for that considering the anisotropic 50 electron scattering.

\section{Conclusions}

The reliable electron collision cross section set of water vapor 54 is estimated by the electron swarm method using Monte 55 Carlo simulation. The cross section set consists of two 56 vibrational excitations, three electron attachments, 26 elec- 57 tronic excitations, six ionizations, and three rotational 58 excitation cross sections and one elastic collision cross 59 section, and the anisotropic electron scattering for elastic and 60 rotational excitation collisions is considered. The electron 61 


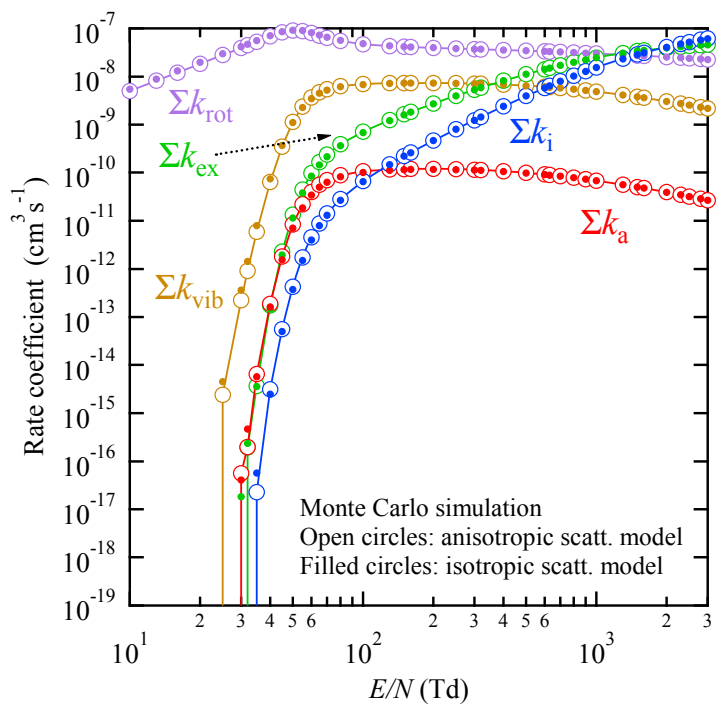

Fig. 12. (Color online) Sum of rate coefficients for inelastic collisions in water vapor.

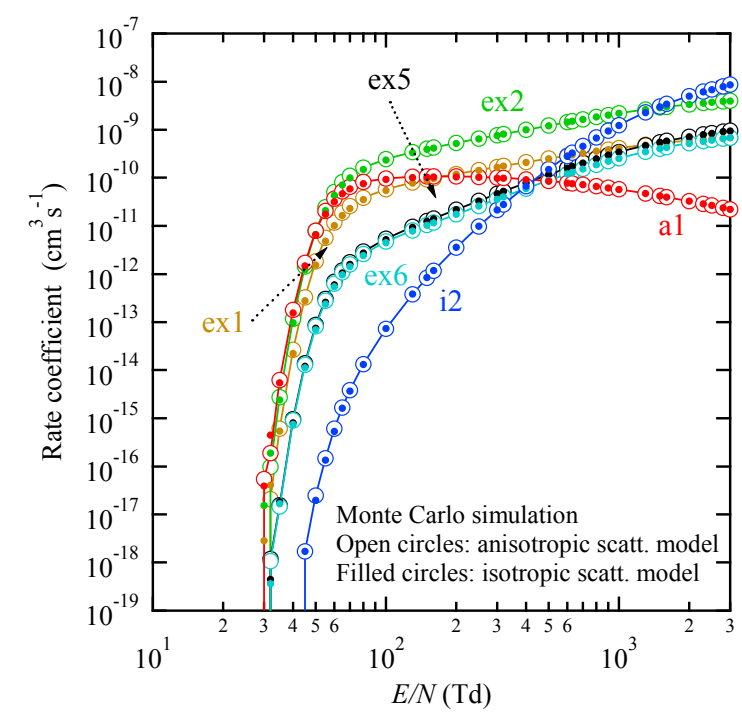

Fig. 13. (Color online) Rate coefficients for the generation of OH radical in water vapor.

43 transport coefficients calculated from the present cross ${ }_{44}$ section set are in good agreement with the measured data 45 in a wide range of $E / N$ values. This confirms the validity of ${ }_{46}$ the present cross section set.

The cross section set for water vapor assuming the 48 isotropic electron scattering is proposed for practical use. 49 The electron transport coefficients and rate coefficients cal5o culated by Monte Carlo simulation using the cross section set 51 assuming the isotropic electron scattering are found to agree 52 well with those calculated from the accurate cross section set ${ }_{3}$ considering the anisotropic electron scattering.

1) J. Takahata, K. Takaki, N. Satta, N. Satta, K. Takahashi, T. Fujio, and Y. Sasaki, Jpn. J. Appl. Phys. 54, 01 AG07 (2015).

2) C. A. J. van Gils, S. Hofmann, B. K. H. L. Boekema, R. Brandenburg, and
P. J. Bruggeman, J. Phys. D 46, 175203 (2013).

3) H. Kuwahata, T. Yamaguchi, R. Ohyama, and A. Ito, Jpn. J. Appl. Phys. 54, 01AG08 (2015).

4) E. Usui, T. Ohshima, H. Yamazaki, S. Ikawa, K. Kitano, N. Maeda, and Y. ${ }^{3}$ Momoi, Nihon Shika Hozongaku Zasshi 58, 101 (2015) [in Japanese].

5) Y. Miyazaki, K. Satoh, and H. Itoh, Electr. Eng. Jpn. 174 [2], 1 (2011).

6) H. Shiota, H. Itabashi, K. Satoh, and H. Itoh, Denki Gakkai Ronbunshi A 132, 297 (2012) [in Japanese].

7) T. Shirafuji and T. Murakami, Jpn. J. Appl. Phys. 54, 01AC03 (2015).

8) D. X. Liu, P. Bruggeman, F. Iza, M. Z. Rong, and M. G. Kong, Plasma Sources Sci. Technol. 19, 025018 (2010).

9) F. Tochikubo, S. Uchida, and T. Watanabe, Jpn. J. Appl. Phys. 43, 315 (2004).

10) Y. Itikawa and N. Mason, J. Phys. Chem. Ref. Data 34, 1 (2005).

11) M. Yousfi and M. D. Benabdessadok, J. Appl. Phys. 80, 6619 (1996).

12) J. de Urquijo, E. Basurto, A. M. Juárez, K. F. Ness, R. E. Robson, M. J. Brunger, and R. D. White, J. Chem. Phys. 141, 014308 (2014).

13) G. Seng and F. Linder, J. Phys. B 9, 2539 (1976).

14) P. Rawat, V. S. Prabhudesai, G. Aravind, M. A. Rahman, and E. Krishnakumar, J. Phys. B 40, 4625 (2007).

15) C. E. Melton, J. Chem. Phys. 57, 4218 (1972).

16) P. A. Thorn, PhD Thesis, School of Chemistry, Physics and Earth Sciences, ${ }^{17}$ Flinders University, Adelaide (2008).

17) M. J. Brunger, P. A. Thorn, L. Campbell, N. Diakomichalis, H. Kato, H. 19 Kawahara, M. Hoshino, H. Tanaka, and Y.-K. Kim, Int. J. Mass Spectrom. 20 271, 80 (2008)

18) P. A. Thorn, M. J. Brunger, H. Kato, M. Hoshino, and H. Tanaka, J. Phys. B ${ }^{21}$ 40, 697 (2007).

19) P. A. Thorn, M. J. Brunger, P. J. O. Teubner, N. Diakomichalis, T. Maddern, M. A. Bolorizadeh, W. R. Newell, H. Kato, M. Hoshino, H. 24 Tanaka, H. Cho, and Y.-K. Kim, J. Chem. Phys. 126, 064306 (2007).

20) P. Thorn, L. Campbell, and M. Brunger, PMC Phys. B 2, 1 (2009).

21) B. G. Lindsay and M. A. Mangan, in Photon and Electron Interactions with 26 Atoms, Molecules and Ions, ed. Y. Itikawa (Springer, New York, 2003) 27 Vol. I/17C, Chap. 5.

22) H. C. Straub, B. G. Lindsay, K. A. Smith, and R. F. Stebbings, J. Chem. ${ }^{28}$ Phys. 108, 109 (1998).

23) K. B. Snow and T. F. Thomas, Int. J. Mass Spectrom. Ion Processes 96, 4930 (1990).

24) D. Lefaivre and P. Marmet, Can. J. Phys. 56, 1549 (1978).

25) J. D. Morrison and J. C. Traeger, Int. J. Mass Spectrom. Ion Phys. 11, $77^{32}$ (1973).

26) H. Ehrhardt and A. Kresling, Z. Naturforsch. A 22, 2036 (1967) [in German].

27) NIST Chemistry Webbook [http://webbook.nist.gov].

28) A. Faure, J. D. Gorfinkiel, and J. Tennyson, Mon. Not. R. Astron. Soc. 347, ${ }^{36}$ 323 (2004)

29) H. Cho, Y. S. Park, H. Tanaka, and S. J. Buckman, J. Phys. B 37, 625 (2004).

30) S. Kawaguchi, K. Satoh, and H. Itoh, Jpn. J. Appl. Phys. 54, 01AC01 (2015).

31) S. Kawaguchi, K. Satoh, and H. Itoh, Eur. Phys. J. D 68, 100 (2014). 41

32) Z. Lj. Petrović, S. Dujko, D. Marić, G. Malović, Ž. Nikitović, O. Šašić, J. 42 Jovanović, V. Stojanović, and M. Radmilović-Rađenović, J. Phys. D 42, 43 194002 (2009).

33) K. Kondo and H. Tagashira, J. Phys. D 23, 1175 (1990).

34) H. Hasegawa, H. Date, and M. Shimozuma, J. Phys. D 40, 2495 (2007). 45

35) K. Satoh, M. Hataguchi, H. Itoh, Y. Sakai, and H. Tagashira, J. Phys. D 27, 46 1480 (1994).

36) M. Yousfi, A. Hennad, and A. Alkaa, Phys. Rev. E 49, 3264 (1994). 47

37) W. H. Press, S. A. Teukolsky, W. T. Vetterling, and B. P. Flannery, 48 Numerical Recipes (Cambridge University Press, Cambridge, U.K., 2007) 49 3rd ed., p. 364

38) R. E. Robson, Introductory Transport Theory for Charged Particles in Gases (World Scientific, Singapore, 2006) p. 26.

39) M. Yousfi, J. de Urquijo, A. Bekstein, E. Basurto, O. Eichwald, J. L. Hernández-Ávila, M. Benhenni, A. M. Juarez Reyes, and N. Merbahi, Proc. 53 17th Int. Conf. Gas Discharges and Their Applications, 2010, p. 574

40) Y. Terashita, K. Satoh, and H. Itoh, Denki Gakkai Ronbunshi A 134, 454 (2014) [in Japanese].

41) K. F. Ness and R. E. Robson, Phys. Rev. A 38, 1446 (1988). 56

42) G. J. M. Hagelaar and L. C. Pitchford, Plasma Sources Sci. Technol. 14, 57 722 (2005) 7 . , 22 . (1) 政

\section{.}

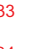
. 38

.
4 . 38 40 